\title{
Sustainicum Collection - Lehrmaterialien für Bildung für nachhaltige Entwicklung
}

richard.kromp@boku.ac.at, Zentrum für Globalen Wandel und Nachhaltigkeit, Universität für Bodenkultur Wien

eingereicht am: 27.12.2015, akzeptiert am: 12.01.2016

Die Sustainicum Collection ist ein offener Pool an Lehrmaterialien zur Veranschaulichung von Grundbegriffen, Prozessen und Zusammenhängen mit starkem Bezug zur Nachhaltigkeit. Die Onlineplattform unterstützt Lehrende inhaltlich und didaktisch bei der Aufgabe, bei Lernenden ein systemisches, ganzheitliches Denken sowie inter- und transdisziplinäre Kompetenzen zu fördern. Die Plattform wurde für die ersten Semester der universitären Lehre entwickelt, eignet sich aber auch für die Sekundarstufe II.

Keywords: Bildung für nachhaltige Entwicklung, vernetztes Denken, Interdisziplinarität, Transdisziplinarität, Schuldbildung

\section{Sustainicum Collection - Teaching materials for education in sustainable development}

The Sustainicum Collection is an open pool of teaching materials to demonstrate basic concepts, processes and interrelations in connection with sustainability. The online platform supports teachers with content and didactically in encouraging students to develop systemic, holistic thinking and inter- as well as transdisciplinary competences. The platform was developed for first-year higher-education teaching but is also suitable for higher secondary education levels.

Keywords: education for sustainable development, holistic approach, interdisciplinarity, transdisciplinarity, secondary school education

\section{$1 \quad$ Sustainicum Collection - Lehrmaterialien für Bildung für nachhaltige Entwicklung}

Sie unterrichten in der Sekundarstufe II und planen, Ihren Unterricht auf nachhaltigkeitsrelevante Themen auszuweiten? Sie haben viel Erfahrung in Ihrem Fachgebiet, aber Sie sind unsicher bei der Auswahl ergänzender Themen und Methoden? Sie würden Ihren Unterricht gerne zukunftsorientierter gestalten, doch die Aufbereitung der Themen nimmt zu viel Zeit in Anspruch? Vielleicht kann Ihnen die Sustainicum Collection bei Ihrem wichtigen Vorhaben ein hilfreiches Werkzeug sein!

Es ist wichtig, Menschen frühzeitig zum nachhaltigen Denken und Handeln anzuregen. Nur so kann der Problematik erfolgreich begegnet werden, dass zahlreiche Lernende trotz Erlangen der Hochschulreife noch wenig Gelegenheit hatten, sich mit nachhaltigen Entwicklungsprozessen mit ökologischer, ökonomischer und sozio-kultureller Bedeutung auseinanderzusetzen und sich im eigenen Leben an Kriterien der Nachhaltigkeit zu orientieren. Um für unsere Nachfahren eine möglichst hohe Lebensqualität zu erhalten, brauchen wir eine nachhaltige Entwicklung in allen Lebensbereichen. Verhaltensmuster sollten so früh wie möglich hinterfragt werden. Eine wichtige Voraussetzung dafür wäre, dass Bildungseinrichtungen dermaßen gestaltet werden, dass Lehrende konsequent ihre Verantwortung für eine nachhaltigkeitsorientierte Bildung wahrnehmen und ihre Rolle in einem breiteren Kontext einer nachhaltigen Entwicklung hin zum Idealbild einer sozial, ökonomisch und ökologisch nachhaltigen Gesellschaft verstehen können (siehe auch IAU 2014; UNECE 2009). Bereits 1997 wurde in der Agenda 21 (BMU 1997) hervorgehoben, dass Bildung eine unerlässliche Voraussetzung für die Förderung einer nachhaltigen Entwicklung und die Verbesserung der Fähigkeit der Menschen sei, sich mit Umwelt- und Entwicklungsfragen auseinanderzusetzen. 


\section{Onlineplattform Sustainicum Collection}

Um eine nachhaltigkeitsorientierte Bewusstseinsbildung zu fördern, wurde eine Onlineplattform für Lehrmaterialien mit Bezug zur Nachhaltigkeit entwickelt (Abb. 1). Die Sustainicum Collection (Plattform Sustainicum 2013) ist unter www.sustainicum. at zugänglich und hat zum Ziel, Lehrenden die Ausweitung ihres Unterrichts auf nachhaltigkeitsrelevante Themen so einfach wie möglich zu machen. Sie können dort nach kleinen „Lehrbausteinen“ suchen, um die eigenen Unterrichtskonzepte mit Aspekten sozialer, ökonomischer und ökologischer Nachhaltigkeit aufzuwerten und Lernende für eine nachhaltige Entwicklung der Gesellschaft besser vorzubereiten. Das kostenlose Angebot an leicht integrierbaren Lehrmaterialien nachhaltigkeitsorientierter Bildung soll dabei helfen, die Vielfalt und Aktualität von Themen der Nachhaltigkeit ebenso wie die Notwendigkeit des persönlichen Engagements für eine lebenswerte Zukunft begreifbar zu machen. Lehrende können die interaktiv gestalteten Ressourcen nutzen, um selbstständiges Arbeiten der Lernenden zu fördern und eine kritische Betrachtung von alltäglichen Vorgängen und einfachen kausalen Zusammenhängen in Gesellschaft und Umwelt aus systemischer und ganzheitlicher Perspektive zu ermöglichen. Die KeywordSuche erleichtert das Auffinden geeigneter Themen. Eine erweiterte Suche ermöglicht auch das Filtern nach bestimmten Kriterien, um gezielt Zeitaufwand, Methoden, Sozialform (Einzel-, Partner- oder Gruppenarbeit), Gruppengröße und vieles mehr zu bestimmen. Dateien wie Videos oder Präsentationsunterlagen können direkt heruntergeladen werden, für die Experimente benötigte Geräte werden beschrieben und können bei Bedarf von den Schulen angeschafft werden. Manche Geräte können auch direkt bei den Autorinnen und Autoren ausgeborgt werden, wobei der Kontakt bei jedem Lehrmaterial angegeben ist.

Eine Stärke der Plattform ist das Sichtbarmachen der Vielfalt an Nachhaltigkeitsthemen. Systemisches und ganzheitliches Denken ist Voraussetzung für ein klares Verständnis für lokale und globale Zusammenhänge und wird durch die Nutzung der Plattform gezielt gefördert. Der Schwerpunkt liegt auf ökologischen und naturwissenschaftlichen Aspekten der Nachhaltigkeit, es wurde aber auch darauf Wert gelegt, dass soziale und ökonomische Aspekte nicht zu kurz kommen. Wie UNECE (2009) feststellte, fokussiert Schulbildung vor allem auf klassische Themen wie Wasserverschmutzung, Müllvermeidung und Energiesparen. Ein interdisziplinärer Ansatz ermöglicht Lernenden, die große Vielfalt an Nachhaltigkeitsthemen fachübergreifend kennenzulernen. Sie werden überrascht sein, wie viele Lehrmaterialien Sie

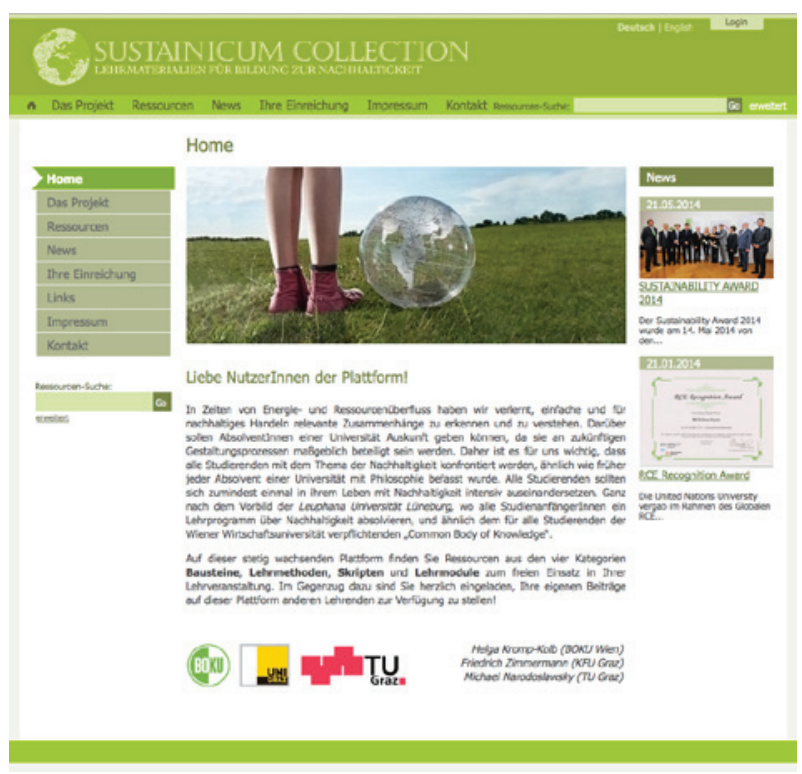

Abb. 1: Screenshot der Startseite der „Sustainicum Collection“

in Ihrem Unterricht einbauen können, die thematisch nicht in Ihrem Fachbereich angesiedelt sind!

Die Sustainicum Collection ist das Ergebnis eines Kooperationsprojekts aus dem Handlungsfeld Bildung für nachhaltige Entwicklung der Universität für Bodenkultur Wien, der Karl-Franzens-Universität Graz und der Technischen Universität Graz. Die drei Universitäten begleiten als Mitglieder der Allianz Nachhaltige Universitäten in Österreich aktiv den aktuellen Prozess zur Förderung von Bildung für nachhaltige Entwicklung im Rahmen des Weltaktionsprogramm Bildung für nachhaltige Entwicklung (2015-2021) als Fortsetzung der UN-Dekade Bildung für nachhaltige Entwicklung (2005-2014).

In einem laufenden Folgeprojekt (ConSus - Science Society Collaboration for Sustainable Development) werden derzeit weitere 90 Lehrmaterialien entwickelt, welche sich mit Transdisziplinarität und Problemstellungen auf lokaler, regionaler und globaler Ebene befassen. Lernende erleben anwendungsorientierte Forschung und wenden das Erlernte in ihrem eigenen Lebensumfeld an. Die neuen Ressourcen werden in Zusammenarbeit von 13 Universitäten aus Österreich, Deutschland, Irland, Albanien und dem Kosovo entwickelt und stehen ab Mitte 2016 zur Verfügung.

\section{Lehrmaterialien}

Die Sustainicum Collection versucht, möglichst viele Aspekte der Nachhaltigkeit zu sammeln. Die einzelnen Lehrmaterialien kann man sich als kleine Bausteine des komplexen Gebäudes vorstellen, welches Bildung für nachhaltige Entwicklung repräsentiert, jedoch kaum vollendet werden kann. Die Sustainicum 


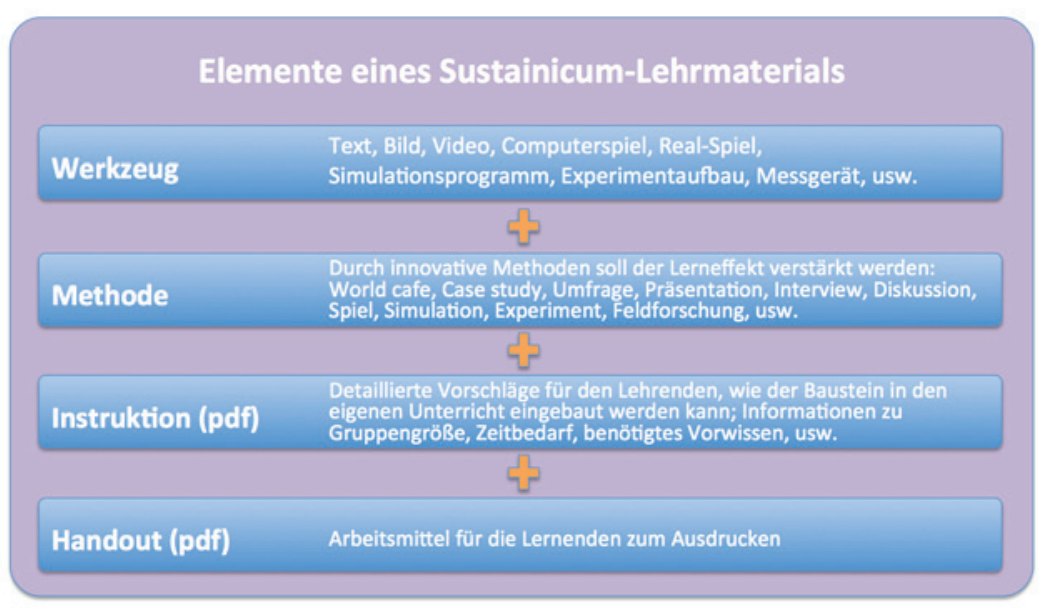

Abb. 2: Jedes Lehrmaterial besteht aus vier wichtigen Elementen.

Collection kann man sich auch als Werkzeugkoffer vorstellen, in dem neben den Werkzeugen und zugehörigen Bedienungsanleitungen auch praktische Anleitungen für Bastelaufgaben enthalten sind.

In Abb. 2 sind die Elemente eines SustainicumLehrmaterials im Überblick dargestellt. Ein Lehrmaterial beinhaltet ein oder mehrere Werkzeuge, wie z. B. Brett- und Computerspiele, Videofilme, einzelne Messinstrumente oder komplette Versuchsanordnungen für einfache naturwissenschaftliche Experimente, Simulationsprogramme oder Präsentationsunterlagen. Der an einem zukunftsorientierten Unterricht interessierte Lehrende findet etwa Kurzfilme über Grundlagen zum Thema Nachhaltigkeit, ein Computerspiel zur nachhaltigen Unternehmensführung, ein Quiz zum Thema Resilienz, ein Experiment zur Erzeugung von Biodiesel, Simulationen zum ökologischen Fußabdruck, ein Rollenspiel zum Thema Philosophie, oder einen unterhaltsamen Animationsfilm über die Gefahren der Nutzung von Atomenergie, um nur einige Beispiele der über 100 Lehrmaterialien zu nennen. Die zugehörige Anleitung für Lehrende beschreibt anschaulich einen konkreten Vorschlag für den Einsatz im Unterricht. Bei der Entwicklung der Unterrichtsmaterialien wurde vorrangig darauf geachtet, dass die Lernenden mittels interaktiver Methoden partizipativ in den Ablauf eingebunden werden. So wird versucht, über die Wissensvermittlung zur Erlangung von Sachkompetenzen hinausgehend auch Selbst- und Sozialkompetenzen für eine nachhaltige Entwicklung der Gesellschaft zu fördern. Dazu zählen je nach Lehrmaterial z. B. individuelle Talente und Fähigkeiten, Wissen um eigene Stärken und Schwächen, Selbstreflexion, Eigeninitiative, Übernahme von Verantwortung, Kooperationsfähigkeit, Partizipation, interdisziplinäre und transdisziplinäre Kompetenzen (Rauch et al. 2008). Mittels service learning werden Lernende dazu animiert, die Lerninhalte in ihrem unmittelbaren Lebensumfeld zu erarbeiten oder auszuprobieren. Beispiele für interaktive Elemente sind Rollenspiele, Fallstudien, kreative
Methoden zum Brainstorming, Strategiespiele, aber auch Simulationen und Experimente. Um den Lerneffekt einer Ressource zu erhöhen, wurde auch darauf geachtet, die Anzahl der Lernziele je Lehrmaterial nach dem Motto „weniger ist mehr" möglichst gering zu halten. Abgerundet wird das Angebot durch Handouts für die Lernenden sowie begleitendes Informationsmaterial, um sich bei Bedarf tiefer in die Thematik einzuarbeiten.

Alle Unterrichtsmaterialien der Sustainicum Collection wurden nach Fertigstellung einem einfachen Evaluierungsprozess unterworfen und auf Benutzerfreundlichkeit, wissenschaftliche Korrektheit und Bezug zur Nachhaltigkeit überprüft.

\section{Ausgewählte Beispiele für Lehrmaterialien}

Der US-amerikanische Ökonom und Autor von Brettspielen zum Thema Nachhaltigkeit Dennis Meadows steuerte der Sustainicum Collection das Lehrmaterial The Tower of Babylon bei. Die Lernenden treten gegeneinander in Kleingruppen an, um aus Bauklötzen Türme zu bauen, wobei der unvermeidliche Konflikt zwischen Resilienz und Effizienz thematisiert wird (vgl. Abb. 3). Unter normalen Umständen nicht leicht erkennbar, wird dieser Konflikt durch dieses Spiel auf unterhaltsame Weise ersichtlich gemacht (Meadows 2013).

Der Sustainicum-Baustein „Landnutzung als Ergebnis sozialer und ökologischer Interaktionen“ veranschaulicht die Zusammenhänge zwischen ökologischen und sozio-ökonomischen Systemen in einer übersichtlichen und stark vereinfachten Form. Die wichtigsten Begriffe für das Verständnis von Systemen und Zusammenhängen werden interaktiv dargestellt und geben den Lernenden die Möglichkeit, die Systeme einzeln und im großen Zusammenhang zu betrachten. Nach Durcharbeiten der Flash-Animation sollen die Studierenden in der Lage sein, zu verstehen, 

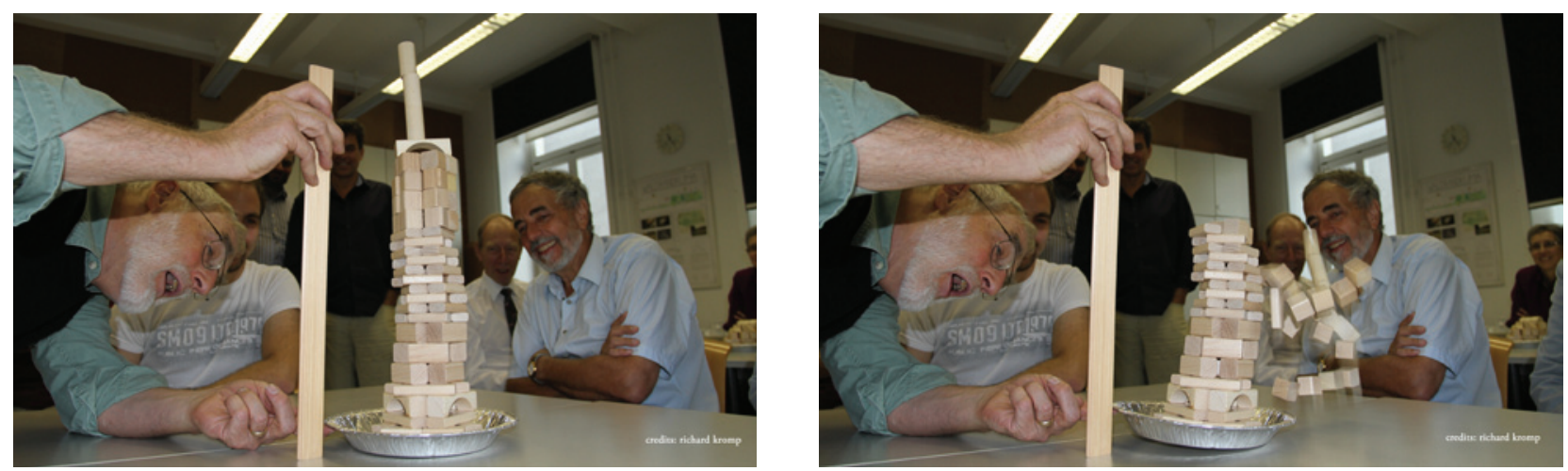

Abb. 3: Links: "The Tower of Babylon “ ist ein unterhaltsamer Turmbauwettbewerb. Rechts: Es gewinnt der Turm mit der kleinsten Größendifferenz vor und nach dem "Tischerdbeben“. (Fotos: Richard Kromp)

dass auf Grund der Komplexität der Interaktionen innerhalb und zwischen den Systemen Lösungen nur dann nachhaltig sind, wenn sie sich auf das gesamte System beziehen. Der Zeitbedarf für den Lehrbaustein wird mit weniger als 30 Minuten angegeben, der Vorbereitungsaufwand für nicht-spezialisierte Lehrende ist niedrig, spezielles Vorwissen der Studierenden wird nicht vorausgesetzt. Für das Arbeiten mit der Flash-Animation wird ein Internet Zugang sowie ein Browser benötigt (Höflehner et al. 2013).

Das Lehrmaterial „Sonne und Schatten - Heliodon" vermittelt die solare Geometrie und deren Wirkung auf Besonnung von Objekten und deren Schattenwirkung. Der Autor stellt fest, dass ein besseres Verständnis der geometrischen Zusammenhänge und der Umgang mit dem Sonnenlicht aus naturwissenschaftlicher Sicht der wichtigste Faktor für nachhaltige Planungen (z. B.: Architektur) ist. Grundlage für den Lehrbaustein ist das Heliodon, ein Sonnensimulator zur anschaulichen Vermittlung der Sonnenbahn im Jahresverlauf für jeden Punkt der Erdoberfläche (vgl. Abb. 4). Auf einem kleinen Drehteller können mit Hilfe von Bauklötzen und Bäumen beliebige Szenarien (z. B. Haus, kleines Dorf) aufgebaut werden und durch Wahl der geographischen Breite und
Hemisphäre der Ort definiert werden. Nun können Bewegungen der Erde um die Sonne und die gegebene Schrägstellung der Drehachse der Erde besprochen werden, und die Schattenbildung des Objekts direkt beobachtet werden. Dem Heliodon ist für Präsentationszwecke eine Web-Videokamera beigefügt, wodurch jedes Szenario durch Projektion einer größeren Anzahl von Lernenden vorgeführt werden kann. Der Zeitbedarf ist kleiner als 30 Minuten und der Vorbereitungsaufwand niedrig, ein Internet Zugang wird nicht benötigt (Mursch-Radlgruber 2013).

\section{Zielgruppe}

Direkte Zielgruppe sind Lehrende der Sekundarstufe II und der ersten Hochschulsemester. Sie können mittels Nutzung der Plattform Ihren Unterricht mit Themen der Nachhaltigkeit ergänzen, oder auf der Plattform eigene Lehrmaterialien hochladen und anderen Kolleginnen und Kollegen zur Verfügung stellen. Mit Hilfe der Lehrmaterialien können neue nachhaltigkeitsrelevante Bildungskonzepte zusammengestellt werden, vom inhaltlichen Detail bis zu großen curricularen Konzepten.
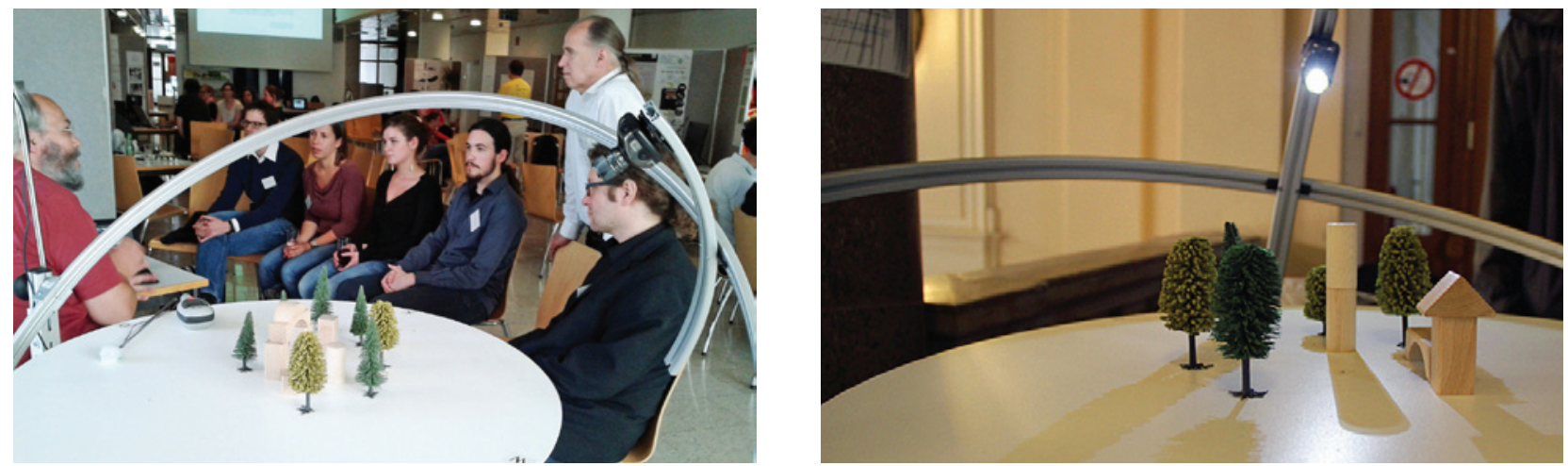

Abb. 4: Links: Sonnensimulator im Rahmen des Lehrmaterials „Sonne und Schatten - Heliodon“. Rechts: Schattenwurf eines Szenarios auf dem Drehteller des "Heliodon". (Fotos: Richard Kromp) 
Indirekte Zielgruppe sind Lernende der Sekundarstufe II und der ersten Hochschulsemester, die durch den Einsatz der Lehrmaterialien eine zukunftsorientierte, interaktive und partizipative Bildung kennenlernen. Neue Aspekte verändern althergebrachte Sichtweisen und bieten Handlungsmöglichkeiten in Richtung einer nachhaltigen Entwicklung.

\section{Akzeptanz der Plattform}

Der Zugang zur Plattform ist online ohne Registrierung auf www.sustainicum.at möglich. Nationale und internationale Netzwerke wie die Allianz Nachhaltige Universitäten in Österreich oder das europäische Hochschulnetzwerk für Bildung für nachhaltige Entwicklung Copernicus Alliance bieten eine gute Voraussetzung für die Verbreitung und Akzeptanz der Sustainicum Collection. Die Plattform wurde auf der "Regional Studies Association European Conference 2013“ in Finnland präsentiert und im Buch „Transformative Wissenschaft - Klimawandel im deutschen Wissenschafts- und Hochschulsystem" (Schneidewind \& Singer-Brodowski 2013) beschrieben. Das Projekt wurde 2013 von der UNESCO-Kommission als „Dekadenprojekt für Bildung für Nachhaltige Entwicklung" gewürdigt. 2014 folgten im Rahmen der RCE International Konferenz in Nairobi der RCE Recognition Award der Kategorie Outstanding Flagship Project und der von den Bundesministerien BMWFW und BMLFUW vergebene Sustainability Award.

\section{$7 \quad$ Literaturverzeichnis}

BMU - Bundesministerium für Umwelt, Naturschutz und Reaktorsicherheit (1997): Agenda 21, Kapitel 36: Förderung der Schulbildung, des öffentlichen Bewusstseins und der beruflichen Aus- und Fortbildung. Kapitel 36.3 + 36.10.d. http://www.agenda21-treffpunkt.de/archiv/ ag21dok/kap36.htm (09.01.2016).

Höflehner, T., M. Kubanek \& M. Gschaider (2013): Landnutzung als Ergebnis sozialer und ökologischer Interaktionen. In: Sustainicum Collection - Lehrmaterialien für Bildung für nachhaltige Entwicklung. http://www. sustainicum.at/de/modules/view/225.Landnutzungals-Ergebnis-sozialer-und-kologischer-Interaktionen (09.01.2016).

IAU - International Association of Universities (2014): Higher education for sustainable development - IAU activities. http://www.iau-aiu.net/content/higher-educationsustainable-development-iau-activities (09.01.2016).

Meadows, D. (2013): The Tower of Babylon. In: Sustainicum Collection - Lehrmaterialien für Bildung für nachhaltige Entwicklung. http://www.sustainicum.at/de/modules/view/186. The-Tower-of-Babylon (09.01.2016).

Mursch-Radlgruber, E. (2013): Sonne und Schatten - Heliodon. In: Sustainicum Collection - Lehrmaterialien für Bildung für nachhaltige Entwicklung. http://www.sustainicum.at/de/modules/view/205.Sonne-und-SchattenHeliodon (09.01.2016).

Plattform Sustainicum (2013): Sustainicum Collection Lehrmaterialien für Bildung für nachhaltige Entwicklung. http://www.sustainicum.at (09.01.2016).

Rauch, F., A. Streissler \& R. Steiner (2008): Kompetenzen für Bildung für Nachhaltige Entwicklung (KOM-BiNE) - Konzepte und Anregungen für die Praxis. https:// www.bmbf.gv.at/schulen/unterricht/ba/bine_kombine_18307.pdf (09.01.2016).

Schneidewind, U. \& M. Singer-Brodowski (2013): Transformative Wissenschaft - Klimawandel im deutschen Wissenschafts- und Hochschulsystem. Marburg, Metropolis Verlag.

UNECE - United Nations Economic Commission for Europe (2009): Learning from each other - The UNECE Strategy for Education for Sustainable Development. B: Sustainable development in formal, non-formal and informal learning. New York und Geneva: UN, 33-34. 